\title{
Diversification of Non-TIR Class NB-LRR Genes in Relation to Whole-Genome Duplication Events in Arabidopsis
}

\author{
Kan Nobuta, ${ }^{1}$ Tom Ashfield, ${ }^{1}$ Sun Kim, ${ }^{2}$ and Roger W. Innes ${ }^{1}$ \\ ${ }^{1}$ Department of Biology and ${ }^{2}$ School of Informatics, Indiana University, Bloomington, IN 47405, U.S.A.
}

Submitted 2 September 2004. Accepted 27 September 2004.

\begin{abstract}
Arabidopsis thaliana is believed to have experienced at least two and possibly three whole-genome duplication events in its evolutionary history. In order to investigate the evolutionary relationships between these duplication events and diversification of disease resistance $(R)$ genes, segmental-duplication events containing $R$ genes belonging to the nucleotide binding-leucine rich repeat (NBLRR) class were identified. Of 153 segmental-duplication events containing NB-LRR genes, only 22 contained NBLRR genes in both members of the duplication pair, indicating a high frequency of NB-LRR gene loss after wholegenome duplication. The relative age of the duplication events was estimated based on the average synonymous substitution rate of the duplicated gene pairs in the segments. These data were combined with phylogenetic analyses. NB-LRR genes present in segment pairs derived from the most recent whole-genome duplication event, estimated to have occurred only 20 to 40 million years ago, occupy very distant branches of the NB-LRR phylogenetic tree. These data suggest that when NB-LRR clusters are duplicated as part of a whole-genome duplication, homoeologous NB-LRR genes are preferentially lost, either by eliminating one copy of the cluster or by eliminating individual genes such that only paralogous NB-LRR genes are maintained.
\end{abstract}

Since the Arabidopsis genome was sequenced completely in 2000 (The Arabidopsis Genome Initiative 2000), it has become an important resource for understanding the origin of segmental duplications and the evolutionary fate of duplicated genes. Simillion and associates (2002) used a software tool for the automatic detection of homologous regions, developed by Vandepoele and associates (2002), to identify duplication events in Arabidopsis. Their analysis of duplicated regions suggested that some regions have been duplicated multiple times. This observation has been shared by other groups, leading to the conclusion that Arabidopsis has gone through multiple rounds of whole-genome duplication events (Bowers et al. 2003; The Arabidopsis Genome Initiative 2000; Lynch and Conery 2000; Simillion et al. 2002; Vision et al. 2000). Three age classes have been identified based on mean $K_{s}$ (synonymous substitution rate) values of the duplicated genes of a segmental-duplication event (Simillion et al. 2002). $K_{s}$ values are calculated using the number of synonymous substitutions per synonymous site between two similar

Corresponding author: R. W. Innes; Telephone: 1-812-855-2219; Fax: 1812-855-6082; E-mail: rinnes@indiana.edu genes and are often used to estimate the date of a gene-duplication event. Simillion and associates (2002) estimated the age classes of the segmental-duplication events at 75,163 , and 221 million years ago, with corresponding mean $K_{s}$ values of $0.91,2.0$, and 2.7. These dates were deduced using an estimated mean rate of synonymous substitutions in Arabidopsis of 6.1 substitutions per $10^{9}$ years (Lynch and Conery 2000). More recent analyses using alternative approaches suggest that these age estimates may be too old. Bowers and associates (2003) estimated the timing of the whole-genome duplication events in the Arabidopsis genome by comparing duplicated genes to gene phylogenies derived from a large number of angiosperms. Using this approach, the most recent whole-genome duplication in Arabidopsis was estimated to have occurred between 15 and 86 million years ago, and the next most recent between 112 and 235 million years ago. Using a slightly different approach, Blanc and associates (2003) estimated the most recent whole-genome duplication to have occurred between 24 and 40 million years ago. Both Bowers and Blanc and their associates (2003) place the most recent event after the divergence of the Arabidopsis lineage from the cotton lineage and before the split between Arabidopsis and Brassica.

After these duplication events occurred, the majority of the duplicated genes were lost, presumably because the duplicates would have had redundant functions (Force et al. 1999; Kashkush et al. 2002; Lynch and Force 2000; Lynch and Conery 2000; Ozkan et al. 2001; Sidow 1996). However, some of the duplicated genes likely escaped elimination by modifying their function or by altering their expression pattern (Force et al. 1999; Lynch and Conery 2000; Lynch and Force 2000; Sidow 1996; Stoltzfus 1999). Approximately 23\% of Arabidopsis genes duplicated in the most recent whole-genome duplication event have been maintained (Blanc et al. 2003), making it possible to identify blocks of duplicated genes, despite large-scale chromosomal rearrangements.

Subsequent to the most recent whole-genome duplication event in Arabidopsis, several gene families expanded further by additional rounds of tandem duplication (Cannon et al. 2004; Leister 2004; Meyers et al. 1999, 2003; Noel et al. 1999). Among these, the nucleotide binding-leucine rich repeat (NB-LRR) gene family is one of the largest and most intensively studied (Cannon et al. 2004; Leister 2004; Meyers et al. 2003; Richly et al. 2002). It is believed that most and perhaps all members of this family function as disease resistance $(R)$ genes, which mediate detection and response to specific pathogens (Dangl and Jones 2001). There are two major classes of NB-LRR genes, TIR (toll interleukin 1 receptor) and nonTIR (Meyers et al. 1999; Pan et al. 2000). The TIR class 
genes have an N-terminal domain that is similar to toll and interleukin 1 receptor proteins, while non-TIR class genes do not have this domain and often contain an N-terminal coiledcoil (CC) domain instead. The TIR and non-TIR classes form distinct clades, even when only the NB domain is used for analysis (Meyers et al. 1999; Pan et al. 2000). This suggests that there has been very little recombination between the two classes (Pan et al. 2000). On the other hand, studies of tandemly repeated NB-LRR genes have revealed that linked genes in the same class recombine frequently (Baumgarten et al. 2003; Meyers et al. 2002, 2003; Noel et al. 1999). Previous studies have also found evidence of segmental-duplication events that include NB-LRR genes (Baumgarten et al. 2003; Richly et al. 2002). These recombination and duplication events are believed to be the driving force for the creation of new $R$ genes, which are necessary to recognize new virulence factors of plant pathogens (Baumgarten et al. 2003; Meyers et al. 2002, 2003; Noel et al. 1999; Parniske et al. 1997).

The evolutionary relationship of Arabidopsis NB-LRR genes that belong to recent duplication events (after the most recent whole-genome duplication event) have been characterized in detail (Baumgarten et al. 2003; Meyers et al. 2003; Noel et al. 1999; Richly et al. 2002). However, the fate of NB-LRR genes after whole-genome duplication events and the evolutionary relationship of NB-LRR genes that belong to old segmental-duplication events are not well understood. In order to investigate these issues, segmental-duplication events containing NB-LRR genes in both members of a duplication pair were identified and the NB-LRR genes belonging to these segmental-duplication events were located on the Arabidopsis NB-LRR gene phylogenetic tree. The correlation between the age of the duplication events and the evolutionary distances among the NB-LRR genes was examined. This analysis revealed an exceptionally high rate of loss of duplicate NB-LRR genes following the most recent wholegenome duplication event. Duplicate NB-LRR gene clusters that have been maintained since this time appear to have lost complementary paralogs, suggesting that redundant NB-LRR genes may be selected against.

\section{RESULTS AND DISCUSSION}

\section{The Arabidopsis genome contains}

many small segmental-duplication events.

In order to examine the evolutionary fate of duplicated genes, Lynch and Conery (2000) identified gene-duplication events in the Arabidopsis genome that involved genes not belonging to large gene families (defined as five or fewer genes with BLAST E values of less than $10^{-10}$ ). The details of their algorithm can be found in Conery and Lynch (2001). They identified 3,881 gene-duplication events. We used these 3,881 geneduplication events as anchor points to define duplicated chromosomal segments. All predicted proteins within $25 \mathrm{~kb}$ of each anchor point were compared using BLASTP. Gene pairs with a BLAST E value of less than $10^{-10}$ within this $25-\mathrm{kb}$ window were considered to be derived from the same duplication event. The BLASTP comparison was then repeated in the adjacent $25 \mathrm{~kb}$ until no more duplication pairs were identified within $25 \mathrm{~kb}$ of the edge of the segment. A $25-\mathrm{kb}$ window was chosen based on the average distance $(10 \pm 15 \mathrm{~kb})$ between the original anchor points. Since the Arabidopsis genome is approximately $117 \mathrm{Mb}$ in size and has 29,084 genes, a 25-kb fragment contains approximately six genes on average. Thus, approximately one in six gene pairs needs to be maintained following a segmental duplication for this algorithm to identify the duplicated segment.
We identified 2,260 segmental-duplication events, including 259 single-gene duplications. In addition to these singletons, 812 segmental-duplication events contained only one gene in one member and many genes in the other member of the duplication pair, suggesting that tandem duplication events occurred within one segment subsequent to the segmental-duplication event or, alternatively, tandem repeats were lost from one of the duplication segments. The largest duplicated segment contained a 492-kb segment of chromosome 1 as one member of the pair and a 707-kb segment of the same chromosome as the other member of the pair. This particular duplicated segment contains 40 gene-duplication events present in the original Lynch and Conery (2000) data set. The two segments encode 131 and 171 genes, respectively. Large intrachromosomal duplication events in the same region of chromosome 1 have been identified by the other groups as well (Blanc et al. 2003; Bowers et al. 2003; The Arabidopsis Genome Initiative 2000; Simillion et al. 2002; Vision et al. 2000). However, the sizes of the duplicated segments are substantially larger than that of the segments found in this study. For example, Simillion and associates (2002) identified duplication segments of 2.29 and 2.00 $\mathrm{Mb}$ containing 584 and 479 genes, respectively, on chromosome 1. Although the largest segmental-duplication pair identified in this study is smaller than that of Simillion and associates, the number of segmental-duplication events is larger.

In order to investigate this discrepancy, the percentage of genes that are covered by segmental-duplication events was examined. The segmental-duplication events identified in this study cover 23,207 genes, which is equivalent to $80 \%(23,207$ genes divided by 29,084 multiplied by 100) of all annotated genes in Arabidopsis. This value is slightly smaller than the values identified by Simillion and associates (2002) (82\%) and Bowers and associates (2003) (89\%) but larger than that identified by Blanc and associates (2003) (71\%). Since these groups used different Arabidopsis genome data sets and a larger window (approximately $100 \mathrm{~kb}$ ) in searching for duplicated genes, it is difficult to perform a direct comparison. Therefore, we merged duplication segments that were less than $100 \mathrm{~kb}$ apart for both pairs, which is similar to the window used by the other groups. After this procedure, the number of segmentalduplication events decreased to 1,956 and coverage increased to $90 \%$ of all genes in the Arabidopsis genome. This result suggests that inversions, deletions, or expansions, singly or in combination, of local regions by tandem duplication have fragmented most large duplicated segments.

\section{Segmental-duplication events with NB-LRR genes can be categorized into two types.}

The complete list of NB-LRR genes in Arabidopsis variety Col-0 was retrieved from The Institute for Genomic Research (TIGR) database (version 3.0), and segmental-duplication pairs containing these genes were identified. There are 193 NB-LRR genes in the TIGR database. Although some of these have been identified as pseudogenes (Meyers et al. 2002, 2003), all were used to identify the segmental-duplication events that contain NB-LRR genes. A total of 149 NB-LRR genes were located in 153 segmental-duplication pairs. These pairs were classified into two basic duplication patterns (Fig. 1). Type I contains NB-LRR genes in both members of the duplication pair, and these NB-LRR gene duplication events are flanked by duplication events of non-NB-LRR genes (Fig. 1). These non-NB-LRR gene duplication events are either on both sides (type Ia) or on only one side (type Ib) of the respective NB-LRR genes. Type II has NB-LRR genes in only one member of the segmental-duplication pair (Fig. 1). In type IIa, there are non-NB-LRR gene duplication events on both sides of the respective NB-LRR genes, whereas type IIb has non-NB-LRR 
gene duplication events on only one side of the respective NBLRR genes (Fig. 1). Together, 11 type Ia, 11 type Ib, 34 type IIa, and 97 type IIb NB-LRR gene segmental-duplication pairs were identified. Thus, at most, only 22 out of 153 duplicated segments maintained NB-LRR genes in both descendents of the duplication.

It is worth noting that none of the duplicated segments have both TIR and non-TIR class NB-LRR genes. In addition, 44 NB-LRR genes did not locate to any segmental-duplication events. These 44 NB-LRR genes included some of the wellcharacterized $R$ genes, such as RPMI (Grant et al. 1995) and RPS2 (Bent et al. 1994). It is possible that the small window size used to identify duplicated segments $(25 \mathrm{~kb})$ missed some NB-LRR genes that should be members of segmentalduplication events. In order to examine this hypothesis, NBLRR genes within $100 \mathrm{~kb}$ from the edge of the identified segmental-duplication events were examined. Surprisingly, no NB-LRR genes were found within this distance. As mentioned above, segmental-duplication events identified using a $100-\mathrm{kb}$ window value covered $90 \%$ of all genes in the Arabidopsis genome, leaving $10 \%$ not associated with a duplication. This value of $10 \%$ is much lower than the percentage of NB-LRR genes not associated with duplications (44 of 193 $=23 \%$ ), suggesting that duplications containing NB-LRR genes may suffer large deletions at a higher rate than the genome as a whole. Consistent with this hypothesis, Cannon and associates (2004) determined that the frequency of NBLRR genes attributable to segmental-duplication events was less than $5 \%$ of the expected frequency.

\section{Analysis of type I duplication events.}

Four out of 22 type I NB-LRR gene segmental-duplication pairs contain non-TIR class NB-LRR genes, while the rest (18 pairs) contain TIR class NB-LRR genes. Figure 2A shows a typical type I NB-LRR gene segmental-duplication (duplication event \#1139), which includes RPS5 and RFL1 (Warren et al. 1998). Note that lines indicating tandem duplications are omitted. The gene-duplication events in black with numbers ( $K_{s}$ values) on the top of the sequence ID are the original six anchor points that we used to identify this segmental-duplication event. In addition to these anchor points, we identified the gene duplications shown in red, green, blue, and black. Red indicates NB-LRR genes and other colors represent non-NBLRR genes. The 10 genes in blue have similarity to a flavinbinding monooxygenase-like protein. Lines connecting these genes have been omitted to simplify this figure. The genes in green have similarity to a protein with PPR repeats (Barkan et al. 1994; Manthey and McEwen 1995). In duplication \#1139, there is only one NB-LRR gene, At1g62630, in one member of the duplication pair (1139B), while there are four NB-LRR genes in the other member (1139A). Almost all the NB-LRR genes in type I events in our analysis showed this duplication pattern (one-to-many). However, Richly and associates (2002) identified recent duplication of some NB-LRR gene clusters in Arabidopsis in which both duplication segments still contain multiple NB-LRR genes (many-to-many). These duplications were not flanked by non-NB-LRR gene duplicates and thus were not identified by our algorithm, which focuses on ancient segmental-duplication events. The lack of many-to-many NBLRR duplications derived from the most recent whole-genome duplication event suggests that either clusters did not exist at the time of the most recent whole-genome duplication or that NB-LRR genes are selectively lost following whole-genome duplications. A similar conclusion was reached by Meyers and associates (2003), who observed that NB-LRR genes were underrepresented in the segmental duplications of Arabidopsis defined by Simillion and associates (2002) and rarely appeared in syntenic positions. Because NB-LRR genes are found in clusters in all angiosperms analyzed to date, selective loss of NB-LRR genes seems far more likely. This conclusion is supported by more global analyses of gene families in Arabidopsis, which have shown that NB-LRR genes are underrepresented relative to other Arabidopsis gene families when comparing either segmental duplications (Cannon et al. 2004) or when looking at gene-pairs believed to be derived from the most recent whole-genome duplication (Blanc and Wolfe 2004).

Since the function of $R$ genes is to recognize the presence of pathogen effector proteins (Dangl and Jones 2001), we speculate that $R$ gene family expansion by duplication may increase the chance that a plant may miss-recognize a self-molecule as foreign, resulting in activation of defense responses. This idea is supported by a report that an interspecific F1 hybrid in genus Nicotiana triggers various defense responses, including programmed cell death (Mino et al. 2002), that are phenotypically similar to those activated by $R$ genes. There should be strong pressure for plants to lose any $R$ genes that autocatalytically trigger cell death, and it is tempting to speculate that this pressure has played a role in the loss of NB-LRR genes in the many-to-many relationship, particularly if the whole-genome duplication was the result of an allopolyploid event (i.e., a cross between different species or subspecies).

\section{Evolutionary relationships among the NB-LRR genes that belong to type I segmental duplications.}

To assess how segmental-duplication events, particularly those associated with whole-genome duplications, have impacted the evolution of NB-LRR gene diversity in Arabidopsis, we located all non-TIR class NB-LRR genes associated with type I duplication events on the well-characterized NB-LRR gene phylogenetic tree (Fig. 3). There are four type I NB-LRR gene segmental-duplication events that have non-TIR class NB-LRR genes (duplication events \#1136, \#1139, \#13134, and $\# 55129$ ). The mean $K_{s}$ values for these duplication events were calculated. The mean $K_{s}$ value of duplication \#55129 is significantly smaller than those of the latest whole-genome duplication event identified by Simillion and associates (2002) and Bowers and associates (2003). This duplication event is a small intrachromosomal duplication and has been previously documented in detail by Meyers and associates (2003). The two NB-LRR genes (At5g43470, At5g48620) belonging to this segmental duplication form a well-supported terminal clade in the phylogenetic tree (Fig. 3), indicating that these arose from an evolutionarily recent event.

Based on the estimated $K_{s}$ values, the other three duplication events are likely the result of the most recent whole-

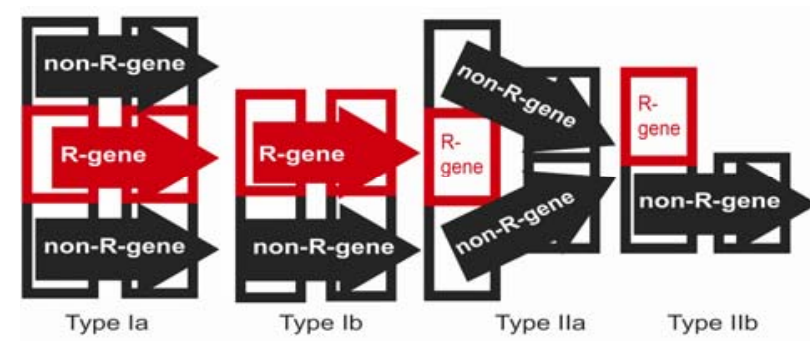

Fig. 1. Two different types of nucleotide binding-leucine rich repeat (NBLRR) gene segmental-duplication events. The black and red blocks indicate the duplication of non-NB-LRR genes and NB-LRR genes, respectively. Type I events have NB-LRR genes in both duplication segments, while Type II events have NB-LRR genes in only one member of the duplication pair. Subtype ' $a$ ' has non-NB-LRR genes on both sides of the NB-LRR gene, whereas subtype ' $b$ ' has non-NB-LRR genes on only one side of the NBLRR gene segmental-duplication event. 
genome duplication. The NB-LRR genes that belong to these duplication events were extracted and located on an NB-LRR gene phylogenetic tree (Fig. 3) (Ashfield et al. 2004). NBLRR genes located within a single segment formed relatively distinct clades (Fig. 3, the green shaded clade). However, the corresponding NB-LRR genes in the paired segments belong to distantly related clades. For example, duplication event \#1136 (Fig. 3, connected with a green line) has one NB-LRR gene (At1g10920) in one segment and four NB-LRR genes in the other segment (At1g61180, At1g61190, At1g61300, At1g61310). These four NB-LRR genes form a well-supported clade in the phylogenetic tree (Fig. 3, green shaded clade), suggesting relatively recent tandem duplications, whereas the NB-LRR gene in the paired segment (At1g10920) belongs to the clade that is connected by the most basal node of the non-TIR tree (Fig. 3, follow the green line). In other words, the ancestor of these NB-LRR genes dates back to the origin of all non-TIR class NB-LRR genes. The mean $K_{s}$ value of this duplication event $(1.2902 \pm 0.752)$ is consistent with it being derived from either the most recent wholegenome duplication or the earlier whole-genome duplication. We therefore compared this duplication segment with the segmental-duplication events identified by Simillion and associates (2002) and Blanc and associates (2003). Both groups placed this duplication segment within a larger duplication block that is clearly derived from the most recent whole-genome duplication event.
Duplication event \#13134 (Fig. 3, blue lines) provides a second example. In one segment are NB-LRR genes At3g14460 and At3g14470, which form a distinct clade by themselves. This clade has a very long branch length, suggesting that At3g14460 and At3g14470 are the result of an ancient tandem duplication event. The duplicated segment contains NB-LRR gene At1g53350. As described above for duplication \#1136, these NB-LRR genes are connected only near the base of the non-TIR tree. The $K_{s}$ value of this duplication event is $0.90 \pm$ 0.16 , suggesting that it results from the most recent wholegenome duplication.

A simple interpretation of the above date would suggest that virtually all of the diversity found within the non-TIR NB-LRR gene family in Arabidopsis arose subsequent to the last wholegenome duplication event. However, we believe this is incorrect. Genes At3g14460 and At1g53350 belong to different basal clades of NB-LRR genes, believed to have originated prior to the monocot/dicot split (Cannon et al. 2002) many millions of years prior to the most recent whole-genome duplication event in Arabidopsis. This paradox can be explained if one assumes that some of the NB-LRR gene clusters in Arabidopsis are quite ancient, with individual genes in these clusters diverging at a steady rate from each other. If such a cluster is duplicated and complementary members of each cluster then subsequently lost from the genome, it would give rise to the appearance of homoeologous genes that have diverged dramatically faster than the genes flanking the cluster. This scenario is supported by close

A

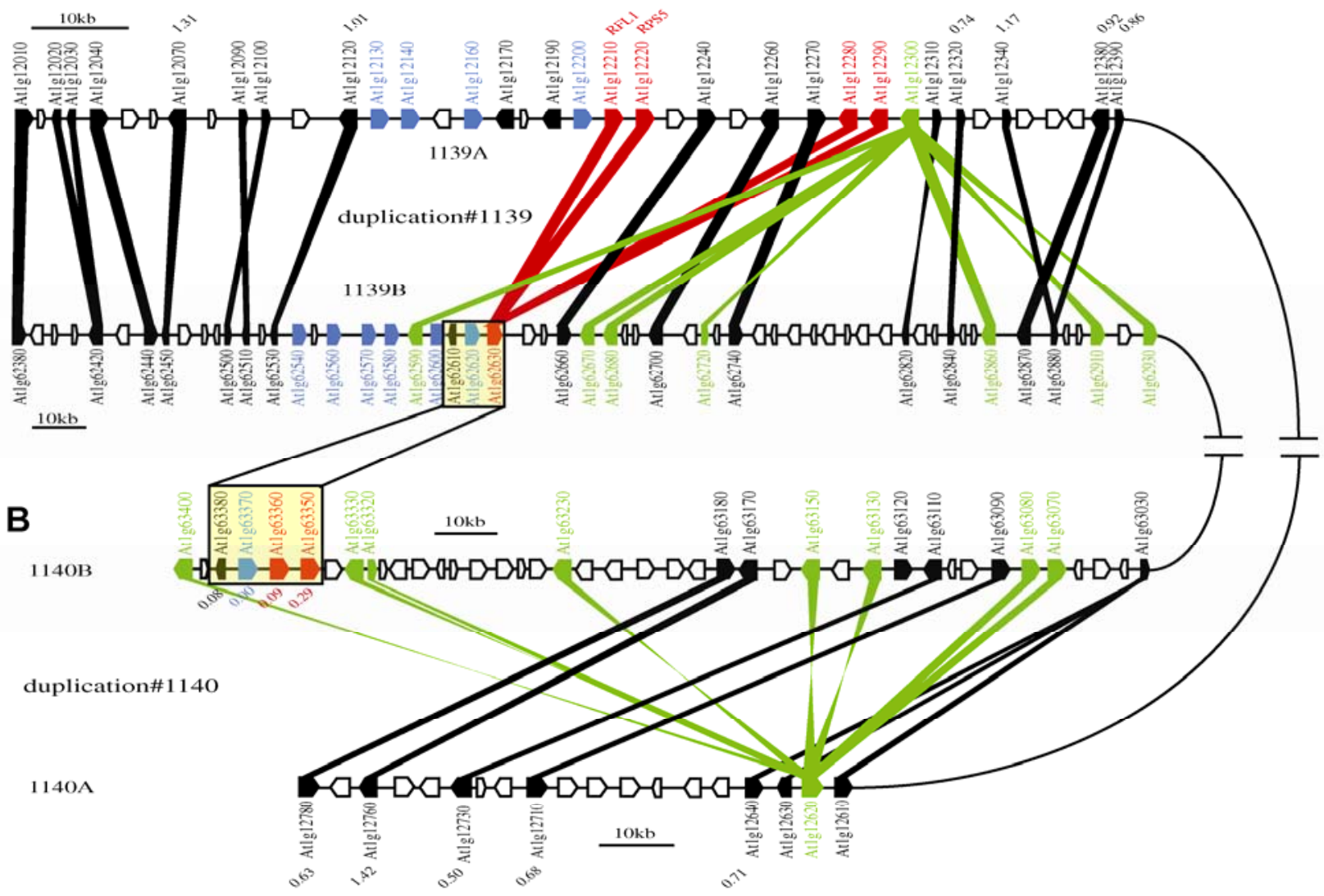

Fig. 2. Example of type I and II nucleotide binding-leucine rich repeat (NB-LRR) gene segmental-duplication events. A, Duplication event \#1139 represents a type I NB-LRR gene segmental-duplication event. B, A type II NB-LRR gene segmental-duplication event that is represented by duplication \#1140. The polygons represent genes, with duplicated genes being identified by their Arabidopsis Genome Initiative (AGI) identification numbers. The numbers above the AGI labels are $K_{s}$ values of the corresponding duplication pairs. The polygons with black $K_{s}$ values are the anchor points that were used to identify segmental-duplication events. Green polygons represent genes that have similarity to a PPR repeat gene. Blue polygons represent genes that have similarity to flavin-binding monooxygenase. Red polygons represent NB-LRR genes. The lines connecting genes indicate homologous genes. Yellow shaded areas represent a recent small segmental-duplication event. Note that the scale of each fragment is different. 
inspection of duplication segment \#13134 (Fig. 4). Gene order is remarkably well conserved among the duplicated genes, except for the NB-LRR genes, which are shifted relative to the flanking genes. This likely reflects that different NB-LRR gene paralogs were lost from each segment. That this cluster is an ancient cluster is also supported by the high level of divergence between genes At3g14460 and At3g14470, despite these genes being located in tandem. In fact, these two genes are more closely related to the soybean Rpgl-b gene than to any other NB-LRR gene in Arabidopsis (Fig. 3; Ashfield et al. 2004), suggesting that they predate the split between legumes and brassicas. We

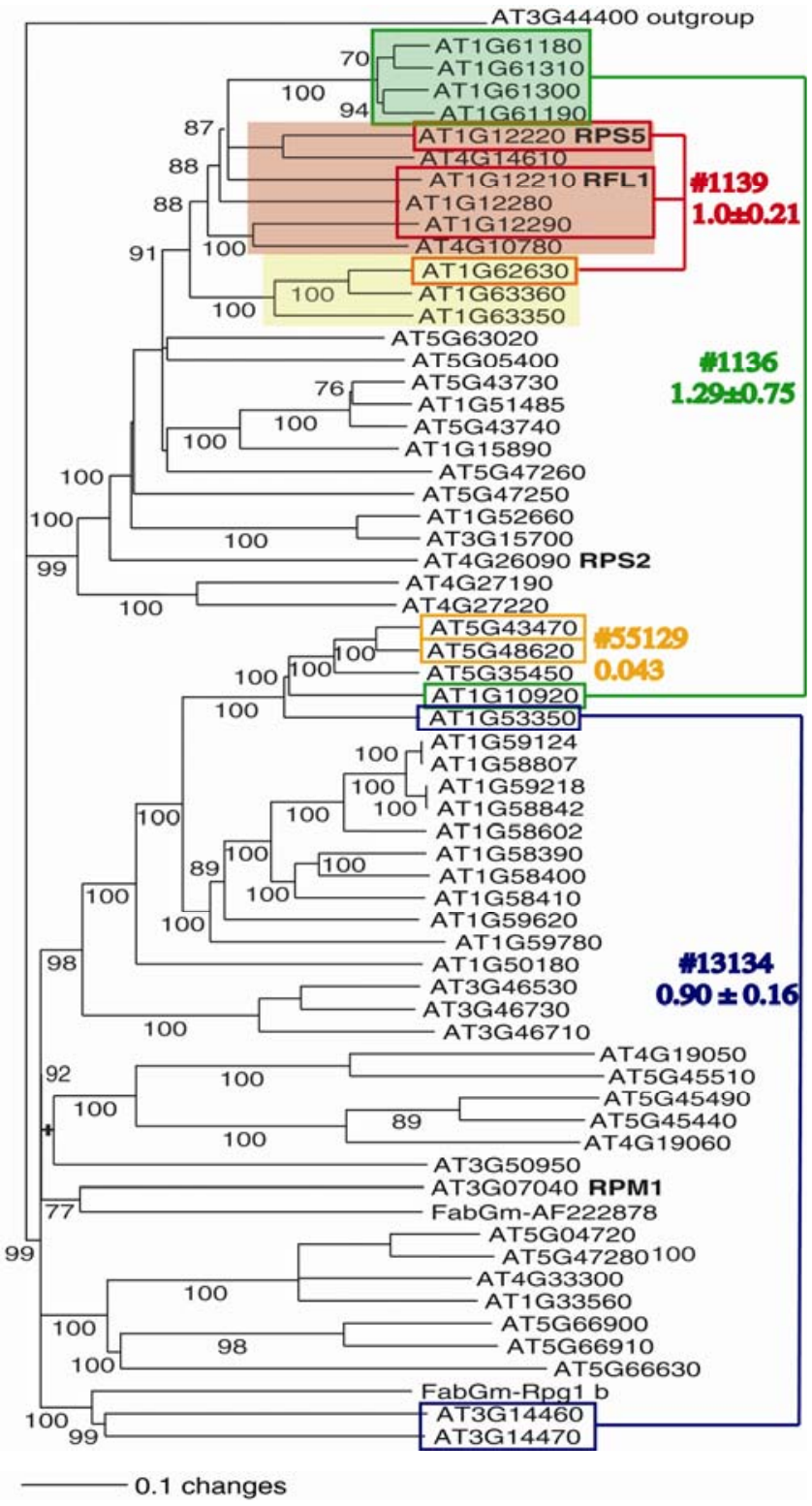

Fig. 3. Evolutionary relationships among the non-toll interleukin 1 receptor (TIR) class nucleotide binding-leucine rich repeat (NB-LRR) genes that belong to type I NB-LRR gene segmental-duplication events. This phylogenetic tree was generated using a Bayesion inference approach and contains the entire set of Arabidopsis non-TIR-class NB-LRR genes, with one TIRclass NB-LRR gene (At3g44400) as the outgroup. The numbers at the interior branches are the percentage of the time that the clade occurred among the sampled trees (i.e., the posterior probability of that clade existing). Only probabilities of $70 \%$ or greater are shown. The NB-LRR genes that belong to four different type I NB-LRR gene segmental-duplication blocks are indicated by different colors: green indicates duplication block \#1136, red indicates duplication block \#1139, blue indicates duplication block \#13134, and orange indicates duplication block \#55129. The numbers below the duplication numbers indicate the mean $K_{s}$ value of the anchor points. thus conclude that some NB-LRR gene clusters in Arabidopsis are quite ancient and that heterogeneous NB-LRR gene clusters (i.e., those containing NB-LRR genes from different clades) (Richly et al. 2002) may have arisen by simple sequence divergence over long evolutionary time periods rather than by recombination events that brought different clusters together.

Analysis of type II NB-LRR gene segmental duplications.

In type II segmental duplications, only one member of the duplication pair contains one or more NB-LRR genes (Figs. 1 and $2 \mathrm{~B}$ ). Such a pattern could be generated when a small seg-

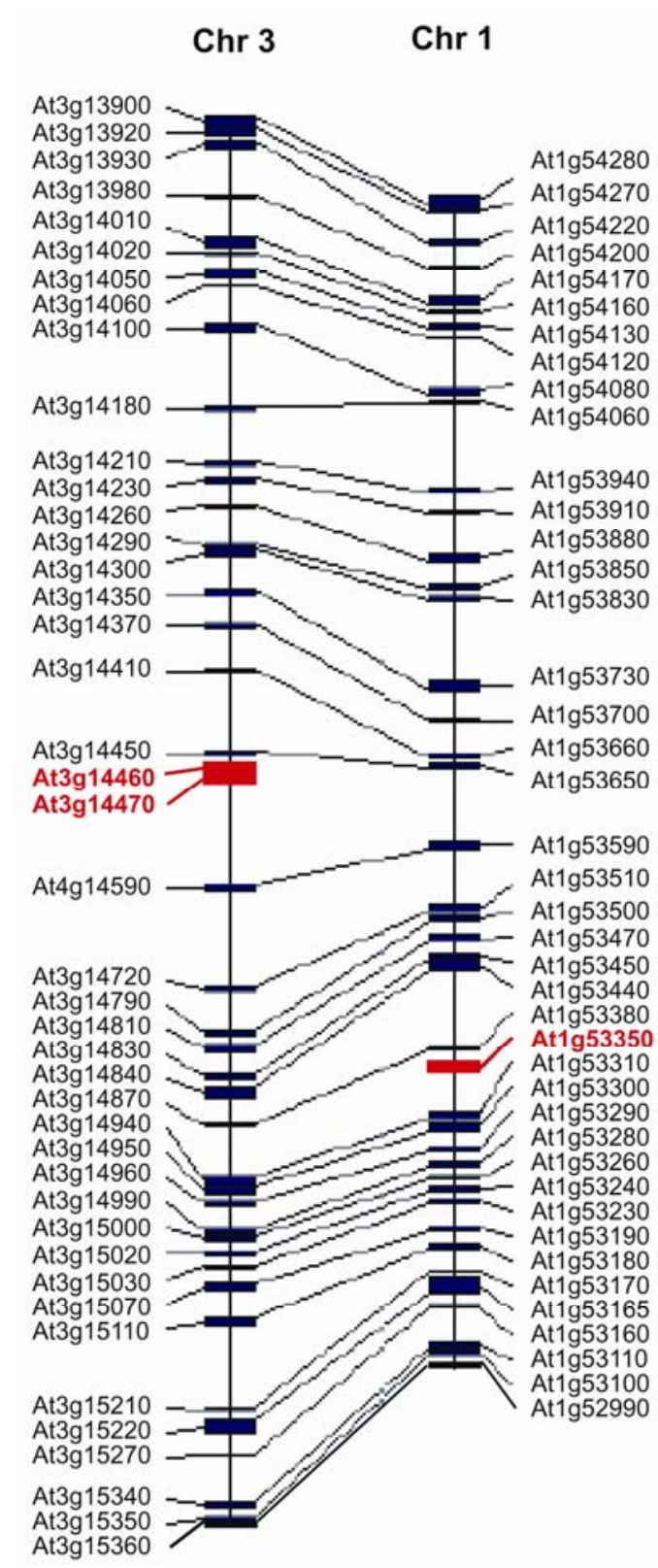

Fig. 4. Loss of complementary nucleotide binding-leucine rich repeat (NB-LRR) gene paralogs from a segmental-duplication event. Duplication block \#13134 shows the relative positions of NB-LRR genes At1g53350 on chromosome 1 and At3g14460 and At3g14470 on chromosome 3 (red blocks). Lines connecting the two chromosomes indicate duplicated genes. Note that the positions of the NB-LRR genes relative to the other genes in the duplication differ, indicating that these represent different paralogs derived from a cluster that existed prior to the whole-genome duplication. Genes are numbered consecutively in units of 10 (e.g., At3g14460 and At3g14470 are adjacent genes). Gaps in sequential numbering represent genes that have not been retained in one of the duplicated segments. 
ment that carries none or more NB-LRR genes inserts into one member of a duplication pair. Duplication event \#1140 strongly supports this scenario. This duplication event has two NB-LRR genes in 1140B but none in 1140A (Fig. 2B). The NB-LRR genes present in 1140B are highly similar to NBLRR gene At1g62630 on duplication segment \#1139B (Fig. 3, yellow shaded clade). Adjacent to these NB-LRR genes are two non-NB-LRR genes (Fig. 2, yellow shaded box). $K_{s}$ values for these non-NB-LRR gene duplications are 0.08 and 0.00 , indicating that this represents a very recent duplication from $1139 \mathrm{~B}$ to $1140 \mathrm{~B}$. The $K_{s}$ values for the anchor genes of duplication 1140 are 0.49 and 0.71 , indicating duplication 1140 arose much earlier, and then, 1140B subsequently acquired a small segmental duplication that contained NB-LRR genes. It is worth noting that segments $1139 \mathrm{~B}$ and $1140 \mathrm{~B}$ are located near each other on chromosome I.

\section{Conclusions.}

Our data indicate that whole-genome duplication events have contributed relatively little to the total number of NBLRR genes present in Arabidopsis today; thus, the great majority of NB-LRR genes must have been lost following whole-genome duplications. Our data do not address whether this loss has occurred at a continuous rate during the 20 to 40 million years since the most recent whole-genome duplication or whether losses occurred rapidly following the duplication event. Comparison of NB-LRR gene clusters among genotypes within the same species typically reveals differences in gene number (Hulbert et al. 2001); thus, the birth and death of NB-LRR genes occurs at a relatively rapid rate. Indeed, many functionally defined $R$ genes are physically absent in susceptible genotypes of the same species (Collins et al. 1999; Grant et al. 1995; McDowell et al. 1998; Noel et al. 1999; Warren et al. 1998). Even a modest benefit on fitness resulting from gene loss should relatively rapidly reduce the number of NB-LRR genes found in the genome. That the loss of an NB-LRR gene can enhance fitness has been demonstrated in the case of the Arabidopsis RPMI gene, which was found to confer a significant fitness cost in field-grown plants when isogenic Arabidopsis lines containing or lacking RPM1 were compared (Tian et al. 2003). Obviously, NBLRR genes must enhance fitness in the presence of appropriate pathogen pressure, but the apparent cost of maintaining these genes likely leads to evolutionarily rapid loss of genes with redundant function, such as would be produced following a whole-genome duplication event.

\section{MATERIALS AND METHODS}

\section{Identification of segmental-duplication events.}

Arabidopsis gene-duplication data were provided by $\mathrm{M}$. Lynch (Indiana University) and J. Conery (University of Oregon, Portland, OR, U.S.A.). This data set contains genes that have five or fewer homologous genes within Arabidopsis with a BLAST E value below $10^{-10}$. In other words, it does not contain large gene families such as the NB-LRR $R$ gene family. Lynch and Conery (2000) identified 3,881 gene-duplication events that contain 5,375 unique genes. These geneduplication events were used as the original anchor points to identify segmental-duplication events. All genes within $25 \mathrm{~kb}$ of each anchor point were then compared by pairwise BLAST analysis to identify additional duplicated genes belonging to each segment, including those belonging to gene families larger than five genes. If additional duplicated genes (BLAST E value $<10^{-10}$ ) were identified, the $25-\mathrm{kb}$ window was moved to the newly defined ends of the segment and the
BLAST analysis was repeated until no more duplicated genes were found.

\section{Determining the relative age of segmental-duplication events.}

The relative ages of segmental-duplication events were estimated using the mean $K_{s}$ values for the genes represented in each duplication. To calculate $K_{s}$ values, the amino acid sequences of duplicated genes were aligned with the CLUSTAL W algorithm (Thompson et al. 1994). The nucleotide sequences corresponding to the aligned amino acid sequences were extracted, and the $K_{s}$ values were calculated using the method developed by Nei and Gojobori (1986).

\section{Identification of Arabidopsis NB-LRR genes.}

S. Salzberg and his group at TIGR have identified 193 NBLRR-related genes in the Arabidopsis genome and categorized them into six groups, based on their domain structures (TIRNB-LRR, CC-NB-LRR, NB-LRR, TIR-NB, CC-NB, TIR). These genes were downloaded from the TIGR database and were used to identify segmental-duplication events that contain NB-LRR genes. In addition to the NB-LRR gene database, the full Arabidopsis genome nucleotide sequence and the amino acid sequence (July 2002 version), also annotated by TIGR, were downloaded. In this database, the total length of the Arabidopsis genome is $117,279,264$ bases and it encodes 29,084 genes.

\section{Phylogenetic analysis.}

The phylogenetic tree presented in Figure 3 was generated using a Bayesian inference method. Amino acid sequences were aligned using ClustalX (Thompson et al. 1997) and the aligned sequences were analyzed using MrBayes v. 2.01 (Huelsenbeck and Ronquist 2001). The analysis shown was run for over 400,000 generations and burn-in was achieved by 50,000 cycles. Trees were sampled every 100 generations. The Dayhoff amino acid substitution model was used. Consensus trees were generated by PAUP* (Sinauer Associates, Sunderland, MA, U.S.A.), using the $50 \%$ majority rule. The numbers at the interior branches are the percentage of the time that the clade occurred among the sampled trees (i.e., the posterior probability of that clade existing). A TIR-NB-LRR class sequence was included as an outgroup.

\section{ACKNOWLEDGMENTS}

We thank M. Lynch and J. Conery for providing the data set of duplicated Arabidopsis genes. This work was supported by National Institutes of Health grant R01 GM46451 to R. W. Innes and National Science Foundation Faculty Early Career Development Program award DBI-0237901 to S. Kim.

\section{LITERATURE CITED}

The Arabidopsis Genome Initiative. 2000. Analysis of the genome sequence of the flowering plant Arabidopsis thaliana. Nature 408:796-815.

Ashfield, T., Ong, L. E., Nobuta, K., Schneider, C. M., and Innes, R. W. 2004. Convergent evolution of disease resistance gene specificity in two flowering plant families. Plant Cell 16:309-318.

Barkan, A., Walker, M., Nolasco, M., and Johnson, D. 1994. A nuclear mutation in maize blocks the processing and translation of several chloroplast mRNAs and provides evidence for the differential translation of alternative mRNA forms. EMBO (Eur. Mol. Biol. Organ.) J. 13:31703181.

Baumgarten, A., Cannon, S., Spangler, R., and May, G. 2003. Genomelevel evolution of resistance genes in Arabidopsis thaliana. Genetics 165:309-319

Bent, A. F., Kunkel, B. N., Dahlbeck, D., Brown, K. L., Schmidt, R., Giraudat, J., Leung, J., and Staskawicz, B. J. 1994. RPS2 of Arabidop- 
sis thaliana: A leucine-rich repeat class of plant disease resistance genes. Science 265:1856-1860.

Blanc, G., and Wolfe, K. H. 2004. Functional divergence of duplicated genes formed by polyploidy during Arabidopsis evolution. Plant Cell 16:1679-1691.

Blanc, G., Hokamp, K., and Wolfe, K. H. 2003. A recent polyploidy superimposed on older large-scale duplications in the Arabidopsis genome. Genome Res. 13:137-144.

Bowers, J. E., Chapman, B. A., Rong, J., and Paterson, A. H. 2003. Unraveling angiosperm genome evolution by phylogenetic analysis of chromosomal duplication events. Nature 422:433-438.

Cannon, S. B., Mitra, A., Andrew, B., Young, N. D., and May, G. 2004 The roles of segmental and tandem gene duplication in the evolution of large gene families in Arabidopsis thaliana. BMC Plant Biol. 4:10.

Cannon, S. B., Zhu, H., Baumgarten, A. M., Spangler, R., May, G., Cook, D. R., and Young, N. D. 2002. Diversity, distribution, and ancient taxonomic relationships within the TIR and non-TIR NBS-LRR resistance gene subfamilies. J. Mol. Evol. 54:548-562.

Collins, N., Drake, J., Ayliffe, M., Sun, Q., Ellis, J., Hulbert, S., and Pryor, T. 1999. Molecular characterization of the maize Rp1-D rust resistance haplotype and its mutants. Plant Cell 11:1365-1376.

Conery, J. S., and Lynch, M. 2001. Nucleotide substitutions and the evolution of duplicate genes. Pacific Symposium of Biocomputing 6:167-178.

Dangl, J. L., and Jones, J. D. 2001. Plant pathogens and integrated defence responses to infection. Nature 411:826-833.

Force, A., Lynch, M., Pickett, F. B., Amores, A., Yan, Y.-1., and Postlethwait, J. 1999. Preservation of duplicate genes by complementary, degenerative mutations. Genetics 151:1531-1545.

Grant, M. R., Godiard, L., Straube, E., Ashfield, T., Lewald, J., Sattler, A., Innes, R. W., and Dangl, J. L. 1995. Structure of the Arabidopsis RPM1 gene enabling dual specificity disease resistance. Science 269:843-846.

Huelsenbeck, J. P., and Ronquist, F. 2001. MRBAYES: Bayesian inference of phylogenetic trees. Bioinformatics 17:754-755.

Hulbert, S. H., Webb, C. A., Smith, S. M., and Sun, Q. 2001. Resistance gene complexes: Evolution and utilization. Annu. Rev. Phytopathol. 39:285-312.

Kashkush, K., Feldman, M., and Levy, A. A. 2002. Gene loss, silencing and activation in a newly synthesized wheat allotetraploid. Genetics 160:1651-1659.

Leister, D. 2004. Tandem and segmental gene duplication and recombination in the evolution of plant disease resistance genes. Trends Genet. 20:116-122.

Lynch, M., and Conery, J. S. 2000. The evolutionary fate and consequences of duplicate genes. Science 290:1151-1155.

Lynch, M., and Force, A. 2000. The probability of duplicate gene preservation by subfunctionalization. Genetics 154:459-473.

Manthey, G. M., and McEwen, J. E. 1995. The product of the nuclear gene PET309 is required for translation of mature mRNA and stability or production of intron-containing RNAs derived from the mitochondrial COX1 locus of Saccharomyces cerevisiae. EMBO (Eur. Mol. Biol. Organ.) J. 14:4031-4043.

McDowell, J. M., Dhandaydham, M., Long, T. A., Aarts, M. G., Goff, S., Holub, E. B., and Dangl, J. L. 1998. Intragenic recombination and diversifying selection contribute to the evolution of downy mildew resistance at the RPP8 locus of Arabidopsis. Plant Cell 10:1861-1874.

Meyers, B. C., Dickerman, A. W., Michelmore, R. W., Sivaramakrishnan, S., Sobral, B. W., and Young, N. D. 1999. Plant disease resistance genes encode members of an ancient and diverse protein family within the nucleotide-binding superfamily. Plant J. 20:317-332.

Meyers, B. C., Morgante, M., and Michelmore, R. W. 2002. TIR-X and TIR-NBS proteins: Two new families related to disease resistance TIRNBS-LRR proteins encoded in Arabidopsis and other plant genomes. Plant J. 32:77-92.

Meyers, B. C., Kozik, A., Griego, A., Kuang, H., and Michelmore, R. W.
2003. Genome-wide analysis of NBS-LRR-encoding genes in Arabidopsis. Plant Cell 15:809-834.

Mino, M., Maekawa, K., Ogawa, K., Yamagishi, H., and Inoue, M. 2002. Cell death processes during expression of hybrid lethality in interspecific $\mathrm{F}(1)$ hybrid between Nicotiana gossei Domin and Nicotiana tabacum. Plant Physiol. 130:1776-1787.

Nei, M., and Gojobori, T. 1986. Simple methods for estimating the numbers of synonymous and nonsynonymous nucleotide substitutions. Mol. Biol. Evol. 3:418-426.

Noel, L., Moores, T. L., van Der Biezen, E. A., Parniske, M., Daniels, M. J., Parker, J. E., and Jones, J. D. 1999. Pronounced intraspecific haplotype divergence at the RPP5 complex disease resistance locus of Arabidopsis. Plant Cell 11:2099-2112.

Ozkan, H., Levy, A. A., and Feldman, M. 2001. Allopolyploidy-induced rapid genome evolution in the wheat (Aegilops-Triticum) group. Plant Cell 13:1725-1747.

Pan, Q., Wendel, J., and Fluhr, R. 2000. Divergent evolution of plant NBSLRR resistance gene homologues in dicot and cereal genomes. J. Mol. Evol. 50:203-213.

Parniske, M., Hammond-Kosack, K. E., Golstein, C., Thomas, C. M., Jones, D. A., Harrison, K., Wulff, B. B., and Jones, J. D. 1997. Novel disease resistance specificities result from sequence exchange between tandemly repeated genes at the $\mathrm{Cf}-4 / 9$ locus of tomato. Cell 91:821-832.

Richly, E., Kurth, J., and Leister, D. 2002. Mode of amplification and reorganization of resistance genes during recent Arabidopsis thaliana evolution. Mol. Biol. Evol. 19:76-84.

Sidow, A. 1996. Gen(om)e duplications in the evolution of early vertebrates. Curr. Opin. Genet. Dev. 6:715-722.

Simillion, C., Vandepoele, K., Van Montagu, M. C., Zabeau, M., and Van de Peer, Y. 2002. The hidden duplication past of Arabidopsis thaliana. Proc. Natl. Acad. Sci. U.S.A. 99:13627-13632.

Stoltzfus, A. 1999. On the possibility of constructive neutral evolution. J. Mol. Evol. 49:169-181.

Thompson, J. D., Gibson, T. J., Plewniak, F., Jeanmougin, F., and Higgins, D. G. 1997. The CLUSTAL X windows interface: Flexible strategies for multiple sequence alignment aided by quality analysis tools. Nucleic Acids Res. 25:4876-4882.

Thompson, J. D., Higgins, D. G., and Gibson, T. J. 1994. CLUSTAL W: Improving the sensitivity of progressive multiple sequence alignment through sequence weighting, position-specific gap penalties and weight matrix choice. Nucleic Acids Res. 22:4673-4680.

Tian, D., Traw, M. B., Chen, J. Q., Kreitman, M., and Bergelson, J. 2003. Fitness costs of $R$-gene-mediated resistance in Arabidopsis thaliana. Nature 423:74-77.

Vandepoele, K., Saeys, Y., Simillion, C., Raes, J., and Van De Peer, Y 2002. The automatic detection of homologous regions (ADHoRe) and its application to microcolinearity between Arabidopsis and rice. Genome Res. 12:1792-1801.

Vision, T. J., Brown, D. G., and Tanksley, S. D. 2000. The origins of genomic duplications in Arabidopsis. Science 290:2114-2117.

Warren, R. F., Henk, A., Mowery, P., Holub, E., and Innes, R. W. 1998. A mutation within the leucine-rich repeat domain of the Arabidopsis disease resistance gene RPS5 partially suppresses multiple bacterial and downy mildew resistance genes. Plant Cell 10:1439-1452.

\section{AUTHOR-RECOMMENDED INTERNET RESOURCES}

Flanders Interuniversity Institute for Biotechnology and Ghent University Plant Systems Biology webpage: www.psb.rug.ac.be

Genetics Department, Trinity College Dublin, Ken Wolfe's lab site for paralogons in Arabidopsis thaliana: wolfe.gen.tcd.ie/athal/dup

The Institute for Genomic Research (TIGR) Arabidopsis thaliana disease resistance gene resource: www.tigr.org/tdb/e2k1/ath1/disRgenes.shtml. 